\title{
Epidemiological changes in infectious diseases during the coronavirus disease 2019 pandemic in Korea: a systematic review
}

\author{
Jong Gyun Ahn, MD, PhD ${ }^{1,2}$ \\ ${ }^{1}$ Department of Pediatrics, Severance Children's Hospital, Yonsei University College of Medicine, Seoul, Korea; ${ }^{2}$ Institute for Immunology and Immunological \\ Diseases, Yonsei University College of Medicine, Seoul, Korea
}

In the era of the coronavirus disease 2019 (COVID-19) pandemic, countries worldwide have implemented several nonpharmaceutical interventions (NPIs) to contain its spread before vaccines and treatments were developed. NPIs included social distancing, mask wearing, intensive contact tracing and isolation, and sanitization. In addition to their effectiveness at preventing the rapid spread of COVID-19, NPIs have caused secondary changes in the epidemiology of other infectious diseases. In Korea, various NPI stages have been implemented since the first confirmed case of COVID-19 on January 20, 2020. This review, based on a PubMed database search, shows the impact of NPIs on several infectious diseases other than severe acute respiratory syndrome coronavirus 2 in the COVID-19 pandemic era in Korea.

Key words: COVID-19, Nonpharmaceutical interventions, Infectious diseases, South Korea

\section{Key message}

- Nonpharmaceutical interventions (NPIs) have had a major impact on the epidemiology of various infectious diseases in Korea.

- Respiratory diseases and gastrointestinal viral diseases were significantly reduced during the NPI period.

- The decrease in Kawasaki disease after the introduction of NPI is an unintended result.

Infectious diseases that decreased during NPI use may reemerge.

- We must continuously monitor the epidemiology of various infectious diseases during the coronavirus era

\section{Introduction}

Since the declaration of the coronavirus disease 2019 (COVID19) pandemic, many countries worldwide, including Korea, have implemented strong quarantine measures based on nonpharmaceutical interventions (NPIs) to suppress its spread. ${ }^{1)}$ NPIs, also called community mitigation strategies, are a set of measures separate from vaccination and medication that help individuals and communities slow the spread of an epidemic. NPIs include social distancing, mask wearing, intensive contact tracing and isolation, and sanitization. Severe acute respiratory syndrome coronavirus 2 (SARS-CoV-2), the virus that causes COVID-19, is a new virus to which humans do not have immunity. Thus, SARS-CoV-2 spread rapidly worldwide and caused a global pandemic. NPIs are one of the best ways to control such pandemics when a vaccine is not yet available. ${ }^{2-4)}$

On February 23, 2020, the Korean government raised the infectious disease crisis level to severe and operated the Central Disaster and Safety Countermeasures Headquarters to focus on pan-government quarantine. Three $\mathrm{T}$ (testing, tracking, treatment) strategies and social distancing adjustments have been made to continuously suppress the incidence of disease and minimize damage in the event of a large-scale outbreak. As of September 2021, the Central Disease Control Headquarters calculates the infection reproduction number $(\mathrm{R})$ to analyze and predict the spread of COVID-19 and applied the quarantine policy in 4 stages according to the $R$ value. ${ }^{5)}$

Since the main transmission routes of SARS-CoV-2 are droplet transmission and direct contact, the NPI policy implemented to suppress its transmission may affect the epidemiologic characteristics of other respiratory and nonrespiratory diseases that are prevalent in the community. In the COVID-19 pandemic, it is important to understand trends in current infectious disease epidemiology and predict the postpandemic epidemiology of infectious diseases. This is because the pediatric population is susceptible to various infectious diseases, and in the current epide. miological situation, a temporary reduction of any infectious disease may lead to a large-scale future outbreak due to the accumulation of susceptible persons.

Therefore, this review discusses epidemiological changes in

Corresponding author: Jong Gyun Ahn, MD, PhD. Department of Pediatrics, Severance Children's Hospital, Yonsei University College of Medicine, 50-1 Yonsei-ro, Seodaemun-gu, Seoul 03722, Korea 


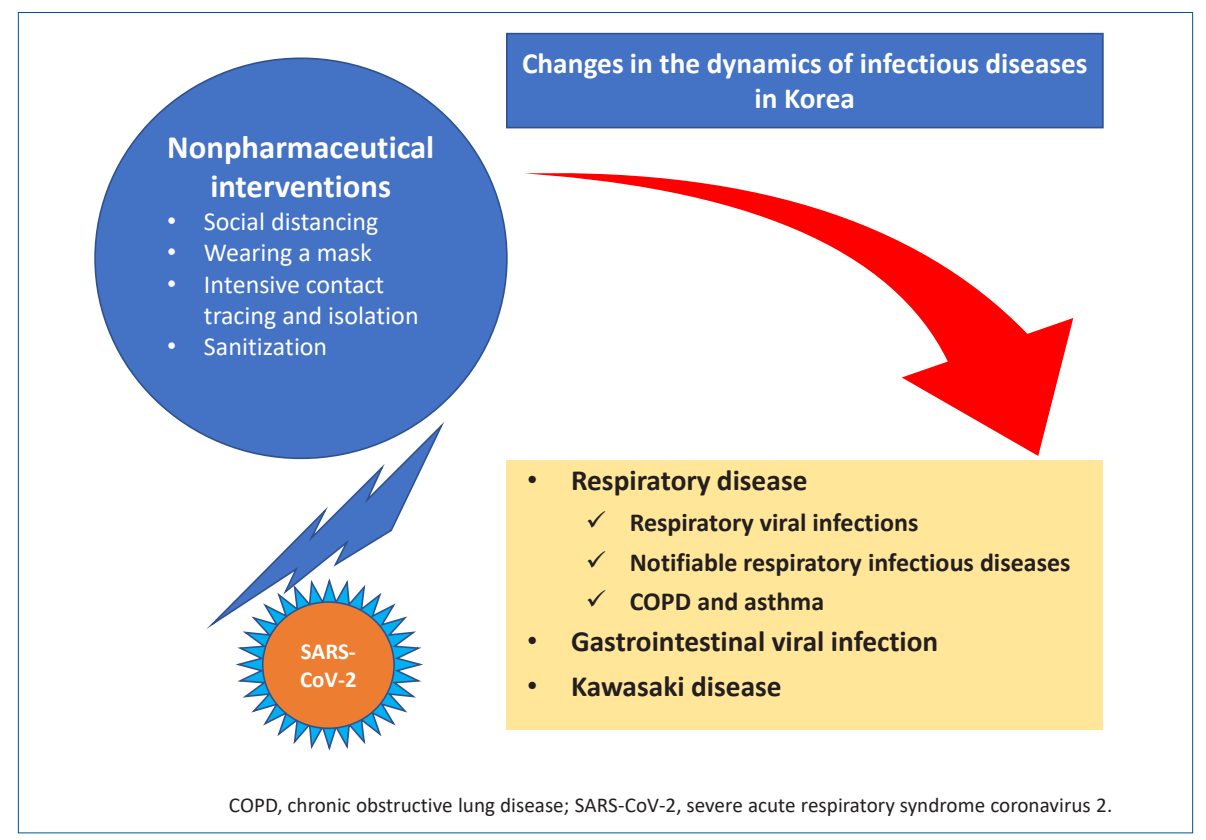

Graphical abstract

infectious diseases during the COVID-19 pandemic in Korea based on searches of the PubMed database. In particular, this review focuses on epidemiologic changes in respiratory and nonrespiratory diseases due to NPIs.

\section{Study selection and characteristics}

PubMed was searched by combining the terms (COVID-19 pandemic, COVID-19, coronavirus disease 2019, SARS-CoV-2, severe acute respiratory syndrome coronavirus 2 , Wuhan coronavirus) and (nonpharmaceutical, social distancing, social distance, cohort grouping, community containment, isolation strategy, isolation, physical contact, physical distancing, quarantine, quarantining, banning, distancing, lockdown, stay at home, contact, mask) and (Korea) to identify studies that reported on epidemiological changes in infectious diseases during the COVID-19 pandemic in Korea. The search was performed on September 24, 2021. The search revealed 699 results; after the removal of duplicates and exclusion of studies based on title and abstract screening, 15 relevant studies were subjected to full-text review. Of them, one was excluded because it was irrelevant to the main topic. Therefore, 14 articles were included in this systematic review (Fig. 1). A summary of the included articles is outlined in Table $1 .^{6-19)}$

\section{Changes in respiratory disease epidemiology}

\section{Respiratory viral infections}

There were a total of 10 studies on respiratory viruses. ${ }^{6-15)}$ Among them, 9 studies were analyzed using data from the surveillance system operated by the Korea Disease Control and

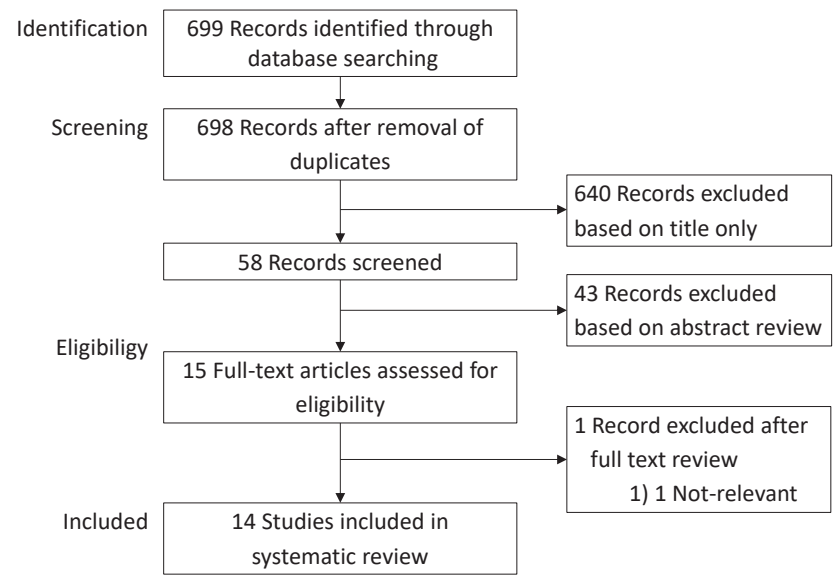

Fig. 1. Flow diagram of study selection process.

Prevention Agency (KDCA), and one study used data obtained from the National Health Insurance Service (NHIS) database. Most studies analyzed the epidemiology of respiratory viral infections during the pandemic compared to the 5 years prior to the COVID-19 pandemic. Four studies ${ }^{8,10,13,14)}$ analyzed all 8 viruses (adenovirus [ADV], parainfluenza virus, respiratory syncytial virus, influenza virus [IFV], human coronavirus, human rhinovirus [HRV], human bocavirus [HBoV], and human metapneumovirus) reported in the surveillance system data, while the other 6 studies $6,7,9,11,12,15)$ analyzed some of the viruses. Of those 6 studies, 4 were of IFV.6,9,11,12) The results differed among studies due to differences in analytical methods and study periods, but all showed that respiratory virus infections after the start of the COVID-19 pandemic had decreasing trends. This is thought to be due to the effectiveness of NPIs used to control COVID-19 infections.

However, 2 studies reported that nonenveloped viruses (ADV, 


\begin{tabular}{|c|c|c|}
\hline Study & Comparison period & Target infections or diseases \\
\hline Huh et al. ${ }^{6)}$ & $\begin{array}{l}\text { January } 2016 \text {-January } \\
2020 \text { vs. February- } \\
\text { July } 2020\end{array}$ & Pneumonia, influenza, COPD, asthma \\
\hline Huh et al.7) & $\begin{array}{l}\text { January } 2016 \text {-January } \\
2020 \text { vs. February- } \\
\text { July } 2020\end{array}$ & $\begin{array}{l}5 \text { Notifiable respiratory diseases (chi- } \\
\text { ckenpox, mumps, invasive pneu- } \\
\text { mococcal disease, scarlet fever, and } \\
\text { pertussis) } \\
\text { Enterovirus } \\
7 \text { Respiratory viruses (ADV, PIV, RSV, } \\
\text { HCoV, HRV, HBoV, and HMPV) } \\
3 \text { Notifiable nonrespiratory infections } \\
\text { (hepatitis A, hepatitis C, carbapenem- } \\
\text { resistant Enterobacterales) }\end{array}$ \\
\hline Kim et al. ${ }^{8)}$ & 2016-2019 vs. 2020 & $\begin{array}{l}8 \text { Respiratory viruses (ADV, PIV, RSV, } \\
\text { IFV, HCOV, HRV, HBoV, and HMPV) }\end{array}$ \\
\hline Kim et al. ${ }^{9)}$ & $\begin{array}{c}\text { January } 2016 \text {-January } \\
2020 \text { vs. February } \\
2020 \text {-January } 2021\end{array}$ & RSV and IFV \\
\hline Kim et al. ${ }^{10)}$ & $\begin{array}{l}\text { 10th-41st weeks of } \\
2015-2019 \text { vs. } 10 \text { th- } \\
\text { 41st weeks of } 2020\end{array}$ & $\begin{array}{l}8 \text { Respiratory viruses (ADV, PIV, RSV, } \\
\text { IFV, HCOV, HRV, HBoV, and HMPV) }\end{array}$ \\
\hline Lee et al. ${ }^{11)}$ & $\begin{array}{l}7 \text { Consecutive seasons: } \\
2013 / 2014 \text { to } 2019 / \\
2020 \text { (each season was } \\
\text { analyzed from week } 36 \\
\text { of the previous year to } \\
\text { week 35, except in } \\
2020 \text { where data were } \\
\text { available up to week } \\
\text { 17) }\end{array}$ & IFV \\
\hline Noh et al. ${ }^{12)}$ & $\begin{array}{l}4 \text { Consecutive seasons: } \\
2016 / 2017 \text { to } 2019 / \\
2020\end{array}$ & IFV \\
\hline
\end{tabular}

Park et al. ${ }^{13)} \quad 2016-2019$ vs. 2020

8 Respiratory viruses (ADV, PIV, RSV, IFV, HCOV, HRV, HBoV, and HMPV)

Park et al. ${ }^{14)}$ 2016-2019 vs. 2020
Data sources Main outcomes

Hospital admission rates after the implementation of NPIs: (1) pneumonia $47 \%$ decrease, (2) influenza 22\% decrease, (3) COPD 58\% decrease, (4) asthma $48 \%$ decrease

KDCA data Observed incidences in the NPI period: (1) respiratory viruses $80.5 \%$ decrease, (2) chickenpox $63.6 \%$ decrease, (3) mumps $36.6 \%$ decrease, (4) invasive pneumococcal disease $43.9 \%$ decrease, (5) scarlet fever $73.9 \%$ decrease, (6) pertussis $70.6 \%$ decrease

Detection rate by year (\%)

(1) Nonenveloped virus: HRV 16.51 vs. 21.42, HBoV 1.95 vs. 3.66, ADV 6.02 vs. 6.20

(2) enveloped virus: IFV 11.46 vs. 4.50, HCoV 4.05 vs. 1.59, HMPV 4.17 vs. 0.70, PIV 6.26 vs. 0.19 , RSV 3.73 vs. 1.45

KDCA data (1) Weekly positive rate of RSV and IFV: $81 \%$ and $94 \%$ reduction for each virus during NPI period

(2) Mean weekly number of hospitalized patients with RSV and IFV: $91 \%$ and $92 \%$ reduction in for each virus during NPI period

KDCA data Mean weekly positive rates: (1) PIV 9.3\% vs $0.1 \%$, (2) IFV $7.2 \%$ vs. $0.1 \%$, (3) HCoV $2.3 \%$ vs $0.4 \%$, (4) HMPV $5.3 \%$ vs. $0.2 \%$

KDCA data ILI in 7 consecutive seasons

(1) early termination of the influenza epidemic in $2019 / 2020$ by $8-12$ weeks compared with previous seasons.

(2) influenza activity peak rate: $49.8 \mathrm{ILIs} / 1000$ visits in 2019/2020 vs. 71.9-86.2 ILIs/1000 visits in previous seasons

(3) influenza hospitalization cases: 161 in 2019/2020 vs. 1914-4327 in previous seasons

(4) influenza B portion: $4.0 \%$ in $2019 / 2020$ vs. $26.6 \%-54.9 \%$ in previous seasons

KDCA data (1) Duration of influenza epidemic period by KDCA: 19 weeks in 2019/2020 vs. 25-31 weeks in previous seasons

(2) Epidemic peak during 2019/2020 season was low in comparison with previous seasons

(1) Peak ILI (cases per 1,000 patients): $2019-2020$ (49.8) vs. 2016-2017 (86.2), 2017-2018 (72.1), and 2018-2019 (73.3)

(2) Yearly positive rates of the respiratory virus during the 8-week postinfluenza period: 2019-2020 (26.5) vs. 2016-2017 (47.7), 2017-2018 (69.9), and 20182019 (67.6)

8 Respiratory viruses (ADV, PIV, RSV, KDCA data Monthly proportion of positive specimens (PPS) relative to the corresponding IFV, HCOV, HRV, HBoV, and HMPV)

(1) Enveloped respiratory viruses: PIV (-88.1\% to $-100 \%)$, RSV (-82.1\% to $-100 \%)$, IFV $(-98.5 \%$ to $-100 \%)$, HCoV $(-52.2 \%$ to $-100 \%)$, and HMPV $(-85.3 \%$ to $-100 \%)$

(2) Nonenveloped viruses: ADV (+76.0\% to $-50.5 \%)$, HRV (+135.8\% to $-72.1 \%)$, and HBoV (+1871.8\% to $-82.6 \%)$

Yun et al. ${ }^{15)} \quad$ 2015-2019 vs. 20206 Vaccine-preventive diseases (hepa(between January and titis $A$, hepatitis $B$, varicella, mumps, June) invasive pneumococcal disease, and pertussis)

Ahn et al. ${ }^{16)} \quad 3$ Consecutive seasons, 2018/2019 to 2020/ 2021 (each season was analyzed from March of the previous year to February)

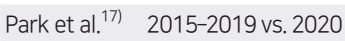

5 Enteric viruses (group A rotavirus, norovirus, sapovirus, astrovirus, and enteric adenovirus)

5 Enteric bacteria (Campylobacter, Clostridium perfringens, nontyphoidal Salmonella, Staphylococcus aureus, and enteropathogenic Escherichia coli)

Norovirus, rotavirus, Campylobacter spp., Clostridium perfringens, and Salmonella spp.
KDCA data Incidences in 2020 to the average of the last 4 years (2015-2019) of the corresponding months.

(1) Decline: $44 \%$ decline for mumps, $44 \%$ decline for varicella, $28 \%$ decline for pertussis, $22 \%$ decline for IPD, $14 \%$ decline in hepatitis $\mathrm{A}$

(2) No change: hepatitis B

KDCA data (1) Reduction rates in 2020/2021 compared to the averages of the last 2 years: total viruses, 31.9\%; norovirus, $40.2 \%$; group A rotavirus, $31.8 \%$; enteric adenovirus, $13.4 \%$; astrovirus, $7.0 \%$; and sapovirus, $12.2 \%$

(2) Incidence of bacterial pathogens 2020/2021 compared to the

- nontyphoidal Salmonella: decreased by $73.0 \%$

- Campylobacter and C. perfringens: increased by $107.9 \%$ and $101.4 \%$, respectively

KDCA data Percentage change in reported cases in 2020 relative to the average number of cases in 2015-2019

(1) Decline: Rotavirus ( $60.8 \%$ ) and norovirus ( $20.5 \%)$

(2) Increase: Campylobacter spp. (+84.4\%), C. perfringens $(+60.4 \%)$, and Salmonella spp. (+3.8\%)

NHIS KD-related hospitalization rate between the 2 periods: (1) similar until April, (2) declined from $-38.8 \%$ (April) to $81.7 \%$ (June)
KD incidence: $31.5 / 100000$ (pre-NPI period) vs. 18.8/100 000 (NPI period)
NHIS

$$
\text { nuary-August) }
$$

Kang et al. ${ }^{19)}$ January 2010-January KD 2020 vs. FebruarySeptember 2020

COPD, chronic obstructive lung disease; NHIS, National Health Insurance Service; NPI, nonpharmaceutical intervention; ADV, adenovirus; PIV, parainfluenza virus; RSV, respiratory syncytial virus; HCoV, human coronavirus; HRV, human rhinovirus; HBoV, human bocavirus; HMPV, human metapneumovirus; KDCA, Korea Disease Control and prevention Agency; IFV, influenza virus; ILI, influenza-like illness; KD, Kawasaki disease; IPD, invasive pneumococcal disease. 
$\mathrm{HRV}$, and $\mathrm{HBoV}$ ) were continuously detected even after the introduction of NPIs. Why the effectiveness of NPIs differed between respiratory viruses remains unclear, but it may be due to the unique characteristics of the specific viruses, subtle differences in respiratory transmission modes and viral shedding durations, and differences in resistance against environmental conditions. ${ }^{8,14,20-22)}$

\section{Notifiable respiratory infectious diseases}

In Korea, the mandatory surveillance of group 1-3 infectious diseases is implemented according to communicable disease types. Two studies ${ }^{7,15)}$ compared the monthly incidence before versus after NPIs for infectious diseases with respiratory transmission monitored by the KDCA. Both studies included varicella, mumps, invasive pneumococcal disease, and pertussis. In one of the studies, scarlet fever was further analyzed.") The monthly incidence of notifiable respiratory diseases during the NPI period decreased remarkably compared to the pre-NPI period. The largest reductions were observed for mumps and chicken pox.

\section{Other respiratory diseases}

One study $\left.{ }^{6}\right)$ compared hospitalization rates before and after NPI for COPD and asthma, which are chronic lung diseases, in addition to pneumonia and influenza. Hospitalization data were collected from the NHIS database. The study results reported that hospitalizations for COPD, asthma, and respiratory infections such as pneumonia and influenza decreased during the NPI era. In this study, the incidence of hospitalization for other acute and chronic diseases, such as diabetic ketoacidosis or hy. perosmolar hyperglycemic state, intracranial hemorrhage, myocardial infarction, and cancer, did not decrease after NPI, which demonstrated that these findings were not attributable to changes in healthcare utilization caused by the COVID-19 pandemic.

\section{Changes in nonrespiratory disease epidemiology}

\section{Gastrointestinal infection}

Two studies ${ }^{16,17)}$ analyzed gastrointestinal infections using open data provided by the KDCA. Common analytical subjects of the 2 studies included norovirus and rotavirus for gastrointestinal viruses and Campylobacter spp., Clostridium perfringens, and nontyphoidal Salmonella for gastrointestinal bacteria. In one study, 3 enteric viruses (sapovirus, astrovirus, and enteric ADV) and 2 enteric bacteria (Staphylococcus aureus and enteropathogenic Escherichia coli) were further analyzed. ${ }^{16)}$

In both studies, the incidence of gastrointestinal viral infection significantly decreased after the introduction of NPI, whereas the incidence of gastrointestinal bacterial infections was not significantly reduced. This is thought to be because, in the case of the former, transmission occurs mainly by direct contact infec- tion, whereas in the case of the latter, transmission is food-borne.

\section{Kawasaki disease}

Kawasaki disease (KD) is an acute febrile vasculitis of childhood with the complication of coronary artery lesions. ${ }^{23)}$ The etiology of KD remains unknown, although its symptoms are thought to be related to an excessive immune system reaction triggered by an infectious agent in patients with genetic susceptibility. ${ }^{24)}$ Many observational epidemiologic studies have provided evidence of a link between $\mathrm{KD}$ and infection. They include clinical similarity to infectious disease in children, geographical and epidemic temporal clusters, seasonal trends, a strong relationship between $\mathrm{KD}$ and infection surveillance, and unusual recurrence. ${ }^{25)} \mathrm{How}$ ever, no single pathogen has been identified to date.

Two studies ${ }^{18,19)}$ analyzed the incidence of KD before and after NPI in Korea using the NHIS claims database. One study ${ }^{19)}$ analyzed the overall KD incidence rate, while another ${ }^{18)}$ analyzed the KD-related hospitalization rate. The incidence of $\mathrm{KD}$ and the KD-related hospitalization rate decreased significantly after the introduction of NPIs. A decrease in KD after NPI has also been reported in the United States, Japan, and Singapore. ${ }^{26}$ These findings emphasize the impact of extrinsic triggers such as common pediatric infections related to NPIs on the occurrence of KD.

\section{Other diseases}

Two studies ${ }^{7,15}$ analyzed notifiable nonrespiratory infections. Both studies included hepatitis A. Hepatitis B was further analyzed in one study, ${ }^{15}$ ) while hepatitis $\mathrm{C}$ and carbapenemresistant Enterobacterales were further investigated in another study.7)

Although the surge in hepatitis A observed in 2019 resulted in a relatively subtle decrease in hepatitis A during the NPI period, the observed cumulative incidence of hepatitis A was within the $95 \%$ confidence interval (CI) of the predicted value. The observed incidence of hepatitis $\mathrm{C}$ and carbapenem-resistant Enterobacterales during the NPI period remained within the 95\% CIs of model predictions. There was also no change in the incidence of hepatitis B during the NPI period versus the pre-NPI period (2015-2019).

\section{Conclusions}

NPIs launched to contain the spread of COVID-19 have had a major impact on the epidemiology of various infectious diseases in Korea. In particular, respiratory diseases and gastrointestinal viral diseases with respiratory transmission and direct contact transmission, the main transmission modes of SARS-CoV-2, were significantly reduced during the NPI period. In addition, the decrease in KD after the introduction of NPI, which was considered a causative factor of these infectious diseases, is an unintended result.

If the policy of responding to COVID-19 changes due to the 
introduction of vaccines and the development of therapeutic agents in the future and Korea transitions to an era "with corona," it is possible that infectious diseases that were reduced during the NPI period will re-emerge. In particular, one or more may appear in the form of an explosive epidemic in groups, including those of children who are susceptible to these infectious diseases. Therefore, it is very important to continuously monitor the epidemiology of these infectious diseases, predict the outbreak of an epidemic, and establish appropriate management policies. In addition, the study of these epidemiological changes may provide clues to the study of the etiology of various immune diseases induced by infectious diseases that occur as unintended consequences. Future research is needed on the immunological pathogenesis of $\mathrm{KD}$ and its relationship to infectious diseases.

\section{Footnotes}

Conflicts of interest: No potential conflict of interest relevant to this article was reported.

Funding: This study was supported by a faculty research grant of Yonsei University College of Medicine (6-2021-0145).

ORCID:

Jong Gyun Ahn@ https://orcid.org/0000-0001-5748-0015

\section{References}

1. Hartley DM, Perencevich EN. Public health interventions for COVID-19: emerging evidence and implications for an evolving public health crisis. JAMA 2020;323:1908-9.

2. Chu DK, Akl EA, Duda S, Solo K, Yaacoub S, Schunemann HJ, et al. Physical distancing, face masks, and eye protection to prevent person-toperson transmission of SARS-CoV-2 and COVID-19: a systematic review and meta-analysis. Lancet 2020;395:1973-87.

3. Fong MW, Gao H, Wong JY, Xiao J, Shiu EYC, Ryu S, et al. Nonpharmaceutical measures for pandemic influenza in nonhealthcare settings-social distancing measures. Emerg Infect Dis 2020;26:976-84.

4. Flaxman S, Mishra S, Gandy A, Unwin HJT, Mellan TA, Coupland H, et al. Estimating the effects of non-pharmaceutical interventions on COVID-19 in Europe. Nature 2020;584:257-61.

5. Coronavirus (COVID-19), Republic of Korea [Internet]. Cheongju (Korea): Korea Disease Control and Prevention Agency; 2021 [cited 2021 Sep 30]. Available from: http://ncov.mohw.go.kr/socdisBoardView. do? brdId $=6 \&$ brdGubun $=1$.

6. Huh K, Kim YE, Ji W, Kim DW, Lee EJ, Kim JH, et al. Decrease in hospital admissions for respiratory diseases during the COVID-19 pandemic: a nationwide claims study. Thorax 2021;76:939-41.

7. Huh K, Jung J, Hong J, Kim M, Ahn JG, Kim JH, et al. Impact of nonpharmaceutical interventions on the incidence of respiratory infections during the coronavirus disease 2019 (COVID-19) outbreak in Korea: a nationwide surveillance study. Clin Infect Dis 2021;72:e184-91.

8. Kim HM, Lee EJ, Lee NJ, Woo SH, Kim JM, Rhee JE, et al. Impact of coronavirus disease 2019 on respiratory surveillance and explanation of high detection rate of human rhinovirus during the pandemic in the Republic of Korea. Influenza Other Respir Viruses 2021;15:721-31.

9. Kim JH, Roh YH, Ahn JG, Kim MY, Huh K, Jung J, et al. Respiratory syncytial virus and influenza epidemics disappearance in Korea during the 2020-2021 season of COVID-19. Int J Infect Dis 2021;110:29-35.

10. Kim MC, Kweon OJ, Lim YK, Choi SH, Chung JW, Lee MK. Impact of social distancing on the spread of common respiratory viruses during the coronavirus disease outbreak. PLoS One 2021;16:e0252963.

11. Lee H, Lee H, Song KH, Kim ES, Park JS, Jung J, et al. Impact of public health interventions on seasonal influenza activity during the COVID-19 outbreak in Korea. Clin Infect Dis 2021;73:e132-40.

12. Noh JY, Seong H, Yoon JG, Song JY, Cheong HJ, Kim WJ. Social distancing against COVID-19: implication for the control of influenza. J Korean Med Sci 2020;35:e182.

13. Park JY, Kim HI, Kim JH, Park S, Hwang YI, Jang SH, et al. Changes in respiratory virus infection trends during the COVID-19 pandemic in South Korea: the effectiveness of public health measures. Korean J Intern Med 2021;36:1157-68.

14. Park S, Michelow IC, Choe YJ. Shifting patterns of respiratory virus activity following social distancing measures for COVID-19 in South Korea. J Infect Dis 2021;224:1900-6.

15. Yun HE, Ryu BY, Choe YJ. Impact of social distancing on incidence of vaccine-preventable diseases, South Korea. J Med Virol 2021;93:1814-6.

16. Ahn SY, Park JY, Lim IS, Chae SA, Yun SW, Lee NM, et al. Changes in the occurrence of Gastrointestinal Infections after COVID-19 in Korea. J Korean Med Sci 2021;36:e180.

17. Park S, Michelow IC, Choe YJ. Trend of gastrointestinal infections following nonpharmaceutical interventions, South Korea, 2020. J Infect Dis 2021;224:368-71.

18. Hwangbo J, Lee JS, Choe SA, Choe YJ. Impact of social distancing on Kawasaki disease-associated hospitalization, South Korea. Pediatr Infect Dis J 2021;40:e383-4.

19. Kang JM, Kim YE, Huh K, Hong J, Kim DW, Kim MY, et al. Reduction in Kawasaki disease after nonpharmaceutical interventions in the COVID-19 era: a nationwide observational study in Korea. Circulation 2021;143:2508-10.

20. Leung NHL. Transmissibility and transmission of respiratory viruses. Nat Rev Microbiol 2021;19:528-45.

21. Lin QY, Lim JYC, Xue K, Yew PYM, Owh C, Chee PL, et al. Sanitizing agents for virus inactivation and disinfection. View (Beijing) 2020 May 24:e16. https://doi.org/10.1002/viw2.16. [Epub].

22. Zlateva KT, de Vries JJ, Coenjaerts FE, van Loon AM, Verheij T, Little $\mathrm{P}$, et al. Prolonged shedding of rhinovirus and re-infection in adults with respiratory tract illness. Eur Respir J 2014;44:169-77.

23. McCrindle BW, Rowley AH, Newburger JW, Burns JC, Bolger AF, Gewitz $\mathrm{M}$, et al. Diagnosis, treatment, and long-term management of Kawasaki disease: a scientific statement for health professionals from the American Heart Association. Circulation 2017;135:e927-99.

24. Ahn JG, Bae Y, Shin D, Nam J, Kim KY, Kim DS. HMGB1 gene poly. morphism is associated with coronary artery lesions and intravenous immunoglobulin resistance in Kawasaki disease. Rheumatology (Oxford) 2019;58:770-5.

25. Kim DS. Kawasaki disease. Yonsei Med J 2006;47:759-72.

26. Shulman ST, Rowley AH. An unintended consequence of pandemic control measures: fewer cases of Kawasaki disease. J Pediatr 2021;239:114.

How to cite this article: Ahn JG. Epidemiological changes in infectious diseases during the coronavirus disease 2019 pandemic in Korea: a systematic review. Clin Exp Pediatr 2022;65:167-71. https://doi.org/10.3345/cep.2021.01515 